\title{
Les interrogatives partielles dans un corpus de théâtre contemporain
}

Florence Lefeuvre

Université Sorbonne Nouvelle

Clesthia EA7345

L'objet de cet article est d'analyser si et en quoi les interrogatives partielles produites dans des textes dramatiques contemporains se différencient des interrogatives partielles produites à l'oral spontané. La notion d' "oral spontané » (productions informelles, élaborées 'en direct') s'oppose à la représentation de l'oral qui est constituée d'une énonciation basée sur un travail antérieur qui passe généralement par de l'écrit; elle est « censée représenter l'oral spontané, mais elle a été élaborée d'avance, et par conséquent elle relève du plan du discours de l'écrit: discours officiels politiques, conférences, sermons religieux, échanges et dialogues de films, dialogues dramaturgiques des pièces de théâtre » (Lefeuvre et Tanguy 2012). Elle se distingue par des «patrons discursifs » (Philippe 2008) ou «des modes de stylisation » (Petitjean 2020), à savoir que «dans l'histoire des genres, certaines constellations de faits de langue $[. .$.$] tendent à se stabiliser au point de former des$ représentations stéréotypées inscrites dans l'imaginaire linguistique d'une époque » (ibidem). Pour pouvoir distinguer la représentation de l'oral de l'oral spontané, nous nous baserons sur des corpus qui ressortent de situations de communication permettant cette comparaison: les pièces de théâtre choisies, tirées du corpus Corpus de Dialogues en Français (CoDiF, Lefeuvre \& Parussa 2020), de Sébastien Thiéry, Jean Dell et Gérald Sibleyras, mettent en scène des personnages de la vie ordinaire, rencontrant une ou des difficultés (l'intrigue ou « nœud» théâtral cf. Scherer $1986:$ 63) qui parviendront à un «dénouement» au fil de la pièce. Nous avons également sélectionné une pièce de Xavier Durringer dont les personnages sont issus d'un milieu modeste voire «populaire » ${ }^{1}$ (un agriculteur qui n'a qu'une télévision en noir et blanc, un homme

${ }^{1}$ Cf. Petitjean 2020. 
issu de l'immigration) pour vérifier si l'on observait des changements importants. Parallèlement, pour l'oral spontané, nous avons choisi des conversations prononcées à bâtons rompus et plus précisément le sous-corpus Repas d'Eslo 2 qui regroupe 22555 mots (cf. Eshkol-Taravella et alii 2012 pour la description de cette enquête sociolinguistique à Orléans). Dans les deux cas, les locuteurs se connaissent (que ce soit dans la vie réelle à l'oral spontané ou bien dans la vie fictive de la représentation théâtrale), se trouvent dans une «situation communicative [...] "familière" » (Kerbrat-Orecchioni 1992) et utilisent une langue de proximité («Sprache der Nähe », Koch et Osterreicher 1985) «propre à un immédiat communicatif » (Koch et Osterreicher 2001). Notre première partie présentera les résultats issus de l'étude du sous-corpus d'Eslo 2 Repas. Dans la deuxième partie nous ciblerons les différences et les ressemblances des résultats tirés du corpus CoDif par rapport à ceux de la première partie. La troisième partie relèvera quelques-uns des paramètres qui peuvent expliquer l'emploi des schémas interrogatifs dans les pièces de théâtre et qui semblent liés à la spécificité du « langage dramatique »(Larthomas 1995).

\section{Les interrogatives partielles à l'oral spontané : vue d'ensemble}

Notre étude sur l'oral spontané se base sur un corpus dans lequel se manifeste un oral informel, correspondant à une pratique non préparée non surveillée du français. Le sous-corpus Repas présente des conversations menées à bâtons rompus entre différents locuteurs se connaissant. Nous avons pu utiliser également le corpus interactionnel «Les appels au 15 » de Luca Greco $^{2}$ ainsi que ponctuellement des résultats issus du corpus scolaire Gomila ${ }^{3}$, du CFPP2000 (Branca et alii 2012), pour affiner certains résultats.

\footnotetext{
${ }^{2}$ Corpus non disponible sur la toile, tiré de la thèse de L. Greco.

${ }^{3}$ Corpus accessible, avec les métadonnées, à l'adresse indiquée dans les références.
} 
Voici ce que donnent les résultats tirés du sous-corpus Eslo 2 dans lequel nous avons répertorié semi-automatiquement, en faisant une recherche systématique par item, 699 interrogatives partielles. Nous présentons également corpus interactionnel de Luca Greco qui nous permettront de mieux évaluer les résultats issus d'Eslo 2 :

\begin{tabular}{|l|l|l|l|l|}
\hline & $\begin{array}{l}\text { Chiffres } \\
\text { bruts } \\
\text { Eslo 2 } \\
\text { sous- } \\
\text { corpus } \\
\text { Repas }\end{array}$ & $\begin{array}{l}\% \\
\text { Eslo 2 } \\
\text { sous- } \\
\text { corpus } \\
\text { Repas }\end{array}$ & $\begin{array}{l}\text { Chiffres } \\
\text { bruts } \\
\text { Corpus } \\
\text { Luca } \\
\text { Greco }\end{array}$ & $\begin{array}{l}\text { Corpus } \\
\text { Luca Greco }\end{array}$ \\
\hline $\begin{array}{l}\text { Interrogatives } \\
\text { canoniques }\end{array}$ & 25 & $4 \%$ & 15 & $15,1 \%$ \\
\hline $\begin{array}{l}\text { Structures en } \text { est- } \\
\text { ce que }\end{array}$ & 57 & $8 \%$ & 7 & $7,1 \%$ \\
\hline Structures clivées & 23 & $3 \%$ & 0 & $0 \%$ \\
\hline $\begin{array}{l}\text { Mot interrogatif } \\
\text { SV }\end{array}$ & 77 & $11 \%$ & 5 & $5 \%$ \\
\hline $\begin{array}{l}\text { Mot interrogatif } \\
\text { in situ (phrases } \\
\text { verbales) }\end{array}$ & 310 & $44 \%$ & 53 & $53,6 \%$ \\
\hline $\begin{array}{l}\text { Interrogatives } \\
\text { averbales }\end{array}$ & 207 & $30 \%$ & 19 & $19,2 \%$ \\
\hline $\begin{array}{l}\text { Total des } \\
\text { interrogatives }\end{array}$ & 699 & $100 \%$ & 99 & $100 \%$ \\
\hline
\end{tabular}

Dans les interrogatives canoniques, nous avons retenu les schémas habituellement cités dans les grammaires de référence (Le Goffic 1993, Wilmet 2003, Riegel et alii 2009) qui comportent l'inversion du sujet clitique :

1. comment fais-tu ces choses-là ? (Eslo 2, Repas)

la postposition du sujet :

2. où est Sereine sur la photo ? (Eslo 2, Repas)

le mot interrogatif sujet :

3. qui veut du pain? (Eslo 2, Repas)

l'infinitif comme noyau de l'interrogative :

4. comment dire? (Eslo 2, Repas) 
On voit qu'elles sont davantage représentées dans le corpus de Luca Greco $(15,1 \%)$ que dans le sous-corpus Repas d'Eslo 2 (4 $\%)$, sans doute en raison d'une plus grande distance de situation entre les locuteurs qui ne se connaissent pas mais également en raison du type d'interactions impliquées par ce corpus : dans ces appels au SAMU, on assiste à une répétition du même schéma interactionnel (quelqu'un appelle, la personne du SAMU répond et pose des questions sur le problème de santé ainsi que sur l'identité des patients), ce qui peut amener à une diversification des schémas interrogatifs ${ }^{4}$.

Nous avons réservé une ligne à part pour les structures en est-ce que, également signalées dans les grammaires de référence, mais qui jouent un rôle particulier dans les pièces de théâtre considérées. Dans le sous-corpus Repas, nous n'avons répertorié que $8 \%$ d'interrogatives partielles en est-ce que et dans le corpus de Luca Greco 7,1\%. Ces résultats sont différents de ceux observés dans d'autres corpus comme le montre la compilation opérée dans Guryev (2017: 115-116): Quillard (2000) signale $22,4 \%$ de questions en est-ce que issues de différents types d'interactions (cf. Guryev, 2017 : 116). Mais les interrogatives averbales ne sont pas retenues dans le chiffrage. Si nous les enlevions de nos résultats, nous obtiendrions $12 \%$ de structures en est-ce que, ce qui est encore loin des pourcentages obtenus dans d'autres corpus. Nous nous en tiendrons aux résultats que nous avons recueillis, le type d'interactions, notamment celui du sous-corpus Repas se rapprochant le plus des dialogues des pièces de théâtre.

Les structures clivées peuvent apparaître, mais dans un pourcentage assez faible (3\% dans le sous-corpus Repas), avec plusieurs schémas possibles :

mot interrogatif c'est que $\mathrm{P}$ :

5. où c'est qu'on éteint (Eslo 2, Repas)

$c^{\prime}$ 'est + mot interrogatif + que P :

6. c'est quoi que tu me sers là? (Eslo 2, Repas)

\footnotetext{
${ }^{4}$ Nous avons constaté la même diversification dans le corpus Gomila, cf. plus bas en 3.2 .
} 
Nous avons intégré dans cet ensemble la structure comportant deux mots en $q u$ - (le mot interrogatif et le «que» propre aux clivées) :

7. il s'amuse là euh comment qu'il s'appelait? (Eslo 2, Repas, 3 occurrences)

ainsi que la structure où le mot que propre aux clivées n'apparaît pas :

8. c'est quoi tu m'avais sorti ? (Eslo 2, Repas, 1 occurrence)

Les interrogatives averbales rassemblent des structures hétérogènes. Elles admettent plusieurs sous-groupes. Il peut d'agir de simples mots interrogatifs :

9. JW992 on devrait plus avoir à payer de taxes alors JW992MAR pourquoi?

JW992 bah parce qu'elle est branchée sur internet (Eslo 2, Repas)

parfois accompagnés de toutes sortes de segments en vue d'une demande d'explication ou de précision :

10. NW958 bah je sais pas je viens de voir [...] une meuf qui est passée et qui est rentrée là-bas

KR001 où là-bas?

ZD520 c'est notre voisine (Eslo 2, Repas)

ou accompagnés de ça (pour l'analyse de ces structures (cf. Lefeuvre 2018) :

11. CT418SOE bah je l'ai vue

CT418 quand ça? (Eslo 2, Repas)

Les énoncés averbaux interrogatifs obéissent rarement, dans ce type de discours, à un schéma binaire (cf. Lefeuvre 2016) «prédicat-support » ou « support-prédicat » comme ici :

12. CT763 alors les petits poulets pour qui? (Eslo 2, Repas, seul exemple trouvé)

Voyons à présent en quoi les dialogues dramatiques se rapprochent de ces schémas interrogatifs lorsqu'ils simulent des échanges pris dans la quotidienneté de différents personnages.

\section{Les interrogatives partielles dans les dialogues dramatiques}

Voici ce que donnent les résultats obtenus dans les pièces de théâtre contemporaines examinées issues du CoDiF : 


\begin{tabular}{|l|l|l|l|l|l|}
\hline & $\begin{array}{l}\text { La } \\
\text { Quille }\end{array}$ & $\begin{array}{l}\text { Un petit jeu } \\
\text { sans } \\
\text { conséquence } \\
2002\end{array}$ & $\begin{array}{l}\text { Cochons } \\
\text { d'Inde }\end{array}$ & $\begin{array}{l}\text { Comme } \\
\text { s'il en } \\
\text { pleuvait } \\
2012\end{array}$ & Momo \\
\hline $\begin{array}{l}\text { Interrogatives } \\
\text { canoniques }\end{array}$ & $5 \%$ & $7 \%$ & $11 \%$ & $6 \%$ & $4 \%$ \\
\hline $\begin{array}{l}\text { Structures } \\
\text { interrogatives } \\
\text { en } \text { est-ce que }\end{array}$ & $40 \%$ & $29 \%$ & $37 \%$ & $30 \%$ & $28 \%$ \\
\hline $\begin{array}{l}\text { Structures } \\
\text { clivées }\end{array}$ & $1 \%$ & $0 \%$ & $0 \%$ & $0 \%$ & $0 \%$ \\
\hline $\begin{array}{l}\text { Mot interrogatif } \\
\text { SV }\end{array}$ & $20 \%$ & $12 \%$ & $11 \%$ & $18 \%$ & $23 \%$ \\
\hline $\begin{array}{l}\text { Structures } \\
\text { interrogatives } \\
\text { in situ }\end{array}$ & $14 \%$ & $21 \%$ & $15 \%$ & $12 \%$ & $18 \%$ \\
\hline $\begin{array}{l}\text { Structures } \\
\text { interrogatives } \\
\text { averbales }\end{array}$ & $20 \%$ & $31 \%$ & $25 \%$ & $34 \%$ & $27 \%$ \\
\hline $\begin{array}{l}\text { Nombre } \\
\text { d'interrogatives } \\
\text { classées }\end{array}$ & 92 & 191 & 169 & 284 & 229 \\
\hline
\end{tabular}

Certains pourcentages semblent similaires à ceux obtenus dans le sous-corpus d'Eslo 2 alors que d'autres s'en différencient nettement.

\subsection{De fortes similitudes dans certains cas}

Les ressemblances relevées concernent 3 schémas interrogatifs.

\subsubsection{Interrogatives canoniques}

Les interrogatives canoniques apparaissent peu dans les pièces analysées. Nous n'avons relevé en effet que très peu d'interrogatives avec inversion du sujet clitique :

13. Laurence: On va lui demander... Hein, faut qu'on lui parle!

André : Comment veux-tu parler avec lui, il est complètement sourd! (CoDiF, Momo) 
Ces dernières apparaissent notamment avec comment et le verbe vouloir. Ce sont des cas de figure que nous avons répertoriés dans le sous-corpus Repas d'Eslo 2 :

14. comment veux-tu t'en sortir? (Eslo 2, Repas, 5 exemples avec vouloir)

En revanche nous n'avons trouvé comment dirais-je dans aucun de ces corpus, ni dans le théâtre contemporain ni à l'oral spontané (16 occurrences dans le CFPP2000 employées en fait comme un marqueur discursif).

\subsubsection{Les structures interrogatives du type "mot interrogatif SV"}

Les résultats tirés des pièces de théâtre sont hétérogènes, certains sont similaires aux pourcentages de l'oral spontané (11 $\%$ dans Eslo 2, sous-corpus Repas) alors que d'autres approchent du double. Quillard (2000) signale 16, $2 \%$ dans différents types d'interactions (cf. Guryev 2017 : 116) alors que nous n'en observons que $5 \%$ dans le corpus de Luca Greco. Mais, si nous les ramenons à un corpus uniquement composé de structures verbales, nous obtenons $15,7 \%$.

La présence de ce schéma apparaît pour des raisons syntaxiques, ce qui pourrait expliquer le peu de différences observées entre les corpus d'oral spontané et les corpus dramatiques. La raison principale semble liée au mot en $q u$ - employé. C'est effectivement l'interrogatif pourquoi qui se trouve en majorité usité dans cette structure ( 22 occurrences sur 52 dans Momo). Ce mot interrogatif est connu pour occuper principalement la position frontale, en raison notamment de sa valeur extraprédicative (cf. Le Goffic 1997) :

15. Pourquoi tu l'écoutes alors? (CoDiF, Un petit jeu sans conséquence)

16. Pourquoi ils nous font ça? (CoDiF, Momo)

Comment est également très régulier (16 occurrences sur 52 dans Momo), peut-être en raison de risques d'ambiguïté pouvant être relevés avec cet interrogatif dans le schéma interrogatif SVQ dans, par exemple, Vous allez comment (cf. Guryev $2017: 74)$.

Cet emploi régulier en position frontale de pourquoi et comment rejoint ce que l'on peut trouver dans les corpus oraux (cf. 
Guryev, 2017 : 119 ; Lefeuvre et Rossi-Gensane 2015). Pour le sous-corpus Repas (Eslo 2), nous n'avons relevé aucun emploi de pourquoi in situ, 57 emplois de comment en position frontale et 5 en position in situ. Cependant comment peut connaitre un emploi in situ (cf. corpus Luca Greco dans lequel on trouve 5 comment en position in situ et 4 comment en position initiale).

Une autre raison peut être avancée pour expliquer la présence de ce schéma, c'est la présence de compléments directs à la droite du verbe, ce qui freine le schéma in situ :

17. Quand tu as du temps de libre? (CoDiF, Momo)

18. Depuis quand tu fréquentes des agents immobiliers? (CoDiF, Momo)

Outre ces similitudes entre l'oral spontané et les pièces de théâtre étudiées, nous verrons en 3 que ce schéma peut être exploité différemment dans ces deux types de discours.

\subsubsection{Les interrogatives partielles averbales}

Nous avons repéré une autre structure récurrente dans les deux types de corpus considérés à l'oral spontané et à l'oral représenté, les interrogatives partielles averbales, avec des résultats comparables, entre 20 et $34 \%$. Tout comme à l'oral spontané, il s'agit rarement d'unités prédicatives à deux termes à part celles qui se constituent du schéma mot interrogatif + pronom ça et que nous analyserons comme un prédicat suivi d'un support qui s'apparente ici à un sujet (cf. Lefeuvre 2018) :

19. Laurence: Tu ne prends pas de ses nouvelles?

Bruno : Comment ça? (CoDiF, Comme s'il en pleuvait)

Nous retrouvons les schémas vus en 1, interrogatives basées sur le seul mot en $q u-$ :

20. Quand? (CoDiF, Comme s'il en pleuvait)

auquelles s'ajoute parfois un élément visant à préciser la demande :

21. Laurence: Depuis on a décidé de ne plus fêter Noël le 25 décembre.

Le Voisin: Je comprends. Et pourquoi le 12 mars? (CoDiF, Comme s'il en pleuvait)

Nous verrons que les différences se situent dans l'emploi de ces structures.

D'autres interrogatives partielles se distinguent nettement, dans leur fréquence, de celles relevées dans les corpus d'oral spontané. 


\subsection{Des disparités}

\subsubsection{Les interrogatives partielles en est-ce que}

La forte fréquence des structures interrogatives en est-ce que dans le théâtre contemporain examiné constitue une première disparité par rapport à l'oral spontané. Cette récurrence de la structure interrogative en est-ce que est manifeste quelle que soit la pièce considérée :

22. Laurence. Et nous qu'est-ce qu'on va manger ce soir? (CoDiF, Momo)

C'est notamment le cas des interrogatives en qu'est-ce qu-, qui se trouvent en concurrence avec la structure in situ en quoi, lorsque la structure permet de cibler un complément d'objet :

23. Laurence : Qu'est-ce qu'il t'a dit ? (CoDiF, Momo)

24. Laurence: Il t'a dit quoi exactement? (CoDiF, Momo)

Mais les interrogatives en qu'est-ce qu- (30 exemples dans Momo, 51 dans Comme s'il en pleuvait, 30 dans La Quille) sont nettement plus récurrentes que celles in situ (5 exemples dans Momo, 10 dans Comme s'il en pleuvait et 2 dans La Quille) alors qu'à l'oral spontané, dans le sous-corpus Repas, ces structures sont employées avec la même fréquence (67 qu'est-ce que visant un complément d'objet et 62 quoi in situ, COD du verbe de l'interrogative).

Cette disparité est encore plus nette avec la structure attributive en qu'est-ce que c'est. Celle-ci est employée plus fréquemment à l'oral représenté qu'à l'oral spontané qui privilégie nettement c'est quoi. Qu'est-ce que c'est apparaît ainsi 5 fois dans Momo, 20 fois dans Comme s'il en pleuvait, 2 fois dans La Quille alors que c'est quoi survient à 9 reprises dans Momo, 5 dans Comme s'il en pleuvait et 2 dans La Quille. Mais, dans Eslo 2, c'est quoi survient à 68 reprises $(2$ occurrences dans le corpus de Luca Greco) alors que qu'est-ce que c'est ne revient que 5 fois $(0$ occurrence dans le corpus de Luca Greco), confirmant d'autres résultats (cf. Lefeuvre 2015).

On voit donc que les interrogatives en qu'est-ce que sont surreprésentées dans le théâtre contemporain par rapport à l'oral spontané.

\subsubsection{Les structures interrogatives in situ}


Les interrogatives in situ, employées régulièrement dans le théâtre contemporain (à la différence du théâtre antérieur, cf. Lefeuvre 2006) sont cependant bien moindres que dans le souscorpus Repas d'Eslo 2, où nous en avons répertorié plus du double, parfois le triple, $45 \%$, chiffre qui monte à $63 \%$ si l'on ne considère que les interrogatives verbales (au-delà des chiffres donnés dans Guryev (2017 : 116), 41, 6 \% dans Quillard (2000) et $57,6 \%$ dans Adli (2015)).

Les structures sont les mêmes d'un corpus à l'autre ; la structure en $c$ 'est est de loin la plus importante :

25. RN488FRE hé mais au fait c'est pas mauvais ton c'est quoi c'était quoi c'était quoi ça (Eslo 2, Repas)

26. ANDRÉ : C'est quoi, ça? (CoDiF, Momo)

Avec un verbe bivalent, l'interrogatif quoi peut correspondre au complément d'objet :

27. JW992PFIL1 et Jacky il a pris quoi ? (Eslo 2, Repas)

28. LE VoISIN : Vous fêtez quoi ? (CoDiF, Comme s'il en pleuvait)

Le mot en qu- in situ survient lorsque le complément direct est un clitique positionné à gauche du verbe :

29. Tu dois le rendre quand? (Eslo 2, Repas)

30. Laurence : Je le mets où ? (CoDiF, Comme s'il en pleuvait) ou le démonstratif ça:

31. BV647 parce que tu appelles ça comment toi (Eslo 2, Repas)

Si l'on retrouve bien dans les dialogues dramatiques les structures interrogatives présentes à l'oral spontané, c'est dans leur fréquence que l'on peut constater des différences, qui s'expliquent par leur usage. Le principe directeur qui amène à l'emploi de tel ou tel schéma interrogatif dans les dialogues de théâtre se trouve dans les ressorts du langage dramatique, tels qu'ils sont décrits par exemple dans Lathomas (1995).

\section{Exploitation des interrogatives partielles à des fins dramaturgiques}

Plusieurs raisons peuvent expliquer le choix de telle ou telle structure interrogative dans le théâtre contemporain. La raison essentielle est la «concentration des effets » expliquée dans Larthomas (1995) par «tout élément qui, dans un dialogue [...] se révèle utile, d'une manière ou d'une autre, parce que susceptible d'amener une réaction, si faible soit-elle, du 
spectateur » (Larthomas, 1995 : 281). Les interrogatives sont un outil particulièrement efficace pour susciter des effets. Nous allons voir ci-dessous quels types d'effets les interrogatives peuvent produire.

\subsection{Enchaînement des répliques}

Pour Larthomas (1995 : 268), «l'interrogation est l'élément enchaînant par excellence ».

Les interrogatives partielles averbales facilitent l'enchaînement des répliques et l'obtention d'un dialogue chargé en apport informationnel :

32. Le client. Si tu as besoin d'argent, je t'en donne, c'est tout.

Le guichetier. Pourquoi?

Le client. Par amitié.

Le guichetier. Quel rapport?

Le client. J'ai de l'argent... t'en as pas... On est copains (CoDiF, Cochons d'Inde)

Ce type d'interrogatives, qui correspond à des demandes d'information, permet d'avoir un texte resserré (cf. Lefeuvre et Tanguy 2012), comme souvent dans le théâtre (Larthomas 1995). L'exemple suivant montre comme précédemment que les questions d'un personnage poussent l'interlocuteur à livrer sa pensée :

33. Patrick: Moi aussi j’ai travaillé avec des Suédois.

Serge : Quel rapport?

Patrick: Ben, c'est le nord! J'étais dans la finance avec eux. Mais c'est fini.

Serge : Vous faites quoi maintenant?

Patrick: Je suis plutôt sur des coups.

Serge : Quel genre?

Patrick: [...] (CoDiF, Un petit jeu sans conséquence)

Grâce à ces interrogatives partielles averbales, le texte dramatique gagne en «densité » (Larthomas, 1995 : 284).

Dans cet enchaînement de répliques, les interrogatives in situ ciblent l'information manquante qui facilitera la poursuite du dialogue. On peut certes trouver de simples demandes d'information, qui ne sont pas forcément liées à une présupposition (comme en (33) avec vous faites quoi, cf. Mathieu 2009) :

34. Il y a quoi par là ? (CoDiF, Un petit jeu sans conséquence) 
35. comment tu t'appelles, Maria, comment, Alvarès, âge, 22, vous habitez où, je sais plus, et vous faites quoi, je sais pas (CoDiF, La Quille)

Mais elles s'appuient surtout sur la phrase assertive précédente, reprenant le même schéma syntaxique et poursuivant « le thème de discours en cours » (cf. Beyssade 2007) tout en focalisant l'attention sur une donnée manquante :

36. Laurence : [...] On va entrer dans son jeu.

André : Mais tu vas entrer comment? (CoDiF, Momo)

37. Laurence : Je vérifie...

Bruno : Tu vérifies quoi ? (CoDiF, Comme s'il en pleuvait)

Ce faisant, elles préservent la continuité thématique du discours et en assurent la progression. Il existe ainsi une tension entre la production d'un discours nouveau et la reprise d'un discours ancien.

Un procédé comparable apparaît avec des interrogatives averbales qui s'appuient sur la phrase précédente en ciblant un élément en particulier. Ici, Une plainte pour quoi permet de préciser le nom plainte de la réplique précédente, employé sans déterminant et qui ne renvoie à aucun référent :

38. Laurence : On aurait dî porter plainte.

André. Une plainte pour quoi ? Vol de Caddie ? (CoDiF, Momo)

Dans un processus régulier, l'interrogatif quoi employé seul porte sur l'actant COD d'un verbe précédent (attend) :

39. SERGENT.- Ce qu'on fait là, c'est une bonne question, on attend, pardi!

MARIA.- Quoi?

SERGENT.- Notre train! (Durringer, La Quille)

Ici, la question porte sur un circonstant de temps :

40. Brigitte : Nous avons été rachetés par un groupe indien... Bank of India.

Le client : Depuis quand?

Brigitte: Bientôt un mois, monsieur Kraft. (CoDiF, Cochons d'Inde)

Les interrogatifs visent à compléter les données lacunaires de la réplique précédente. L'absence de verbe, avec une focalisation portant uniquement sur l'information manquante, resserre le discours dramatique.

\subsection{Demande d'éclaircissement}

Les interrogatifs permettent d'éclaircir un élément qui pourrait s'avérer obscur pour le personnage, mais aussi pour le public 
(cf. Larthomas (1995) sur cette situation de communication particulière). Dans les deux exemples ci-dessous, ils suscitent une précision sur le référent d'un pronom qui pourrait être mal identifié, ici le pronom personnel COD le :

41. Le Voisin : (le manteau dans la main) Je le mets où?

Bruno : Quoi?

Le Voisin : Mon manteau, je le mets où ? (CoDiF, Comme s'il en pleuvait)

et là le pronom personnel sujet $i l$ :

42. Laurence : Comment il s'appelle déjà ?

Bruno : Qui?

Laurence : Le cinglé du dessus. (CoDiF, Comme s'il en pleuvait)

Un mot quelconque, comme ici gays, peut faire l'objet d'une demande de précision, à l'aide du mot quoi :

43. BEN.- [...] Je suis sûr qu'il achète et qu'il mate les revues pour gays!

ROGER.- Les quoi?

MARIA.- Les gays. (CoDiF, La Quille)

Plusieurs interrogatives averbales interviennent avec le démonstratif ça qui renvoie à un référent présent dans la réplique antérieure (cf. Lefeuvre 2018) ; en (44) comment prend une valeur méta-énonciative qui pousse l'interlocuteur à reformuler son propos :

44. Serge : Je le croyais, et puis j'ai appris une mauvaise nouvelle.

Claire : Laquelle?

Serge : Bruno et vous n'avez jamais été séparés.

Claire : Comment ça?

Serge : Depuis le début c'est un jeu, Bruno me l'a dit. (CoDiF, Un petit jeu sans conséquence)

Enfin, la variété des schémas interrogatifs du français, relevés par la plupart des auteurs (cf. Guryev (2017) pour un récapitulatif) peut expliquer que, pour une même demande d'information, plusieurs de ces schémas soient utilisés, souvent dans le but de préciser un point du dialogue. Nous n'avons pas remarqué cet emploi dans le sous-corpus Repas d'Eslo 2 mais dans le corpus scolaire Gomila où l'enseignante demande de nouveau un éclaircissement avec un schéma interrogatif différent :

45. M. viens nous montrer le texte qui correspond à la réponse du lundi est-ce que vous êtes d'accord avec Anne-Sophie?

L. oui non 
M. alors Benjamin pourquoi est-ce que tu dis non?

Benjamin parce que il est pas ici il est pas ici le lundi en fait il est ici le lundi

[...]

M. alors je repose la question êtes-vous d'accord avec AnneSophie ? (Corpus scolaire Gomila)

Dans le corpus de Luca Greco, la formulation du même type de questions peut également expliquer l'utilisation de plusieurs schémas interrogatifs pour cibler une demande d'information similaire :

46. Quel âge a-t-elle?

47. Elle a quel âge?

48. Quel âge? (ex. tirés du corpus de Luca Greco)

Dans le corpus théâtral, l'utilisation de plusieurs schémas interrogatifs peut s'expliquer par l'incompréhension d'un des personnages à propos d'une demande qui lui paraît incongrue :

49. Le client : (il se dirige vers la porte) Laissez-moi sortir !

Brigitte: Qu'est-ce que vous allez faire dehors?

Le client : Pardon?

Brigitte : Vous allez faire quoi dehors ? (CoDiF, Cochons d'Inde)

ou au cas où la réponse serait insuffisante :

50. André : [...] Je suis pas un de tes employés.

Laurence : (piquée à vif) Qu'est-ce que ça veut dire, ça?

André : (s'écrasant) Rien, je veux rien dire...

Laurence : (violente) André, ça veut dire quoi?

André : Ça veut dire que tu ne me fais pas peur, voilà ! (CoDiF, Momo)

voire absente :

51. André: C'est insensé. Mais qu'est-ce que vous me voulez à la fin? (Les deux tirent sur le Caddie). Vous voulez quoi, bordel? (CoDiF, Momo)

\subsection{Procédés de mise en relief}

Pour favoriser la concentration des effets, les interrogatives peuvent être utilisées pour organiser les multiples informations que délivre le texte théâtral et mettre en relief certaines d'entre elles.

Un des premiers procédés peut être liée à la répétition de l'interrogative dans un schéma différent, comme nous l'avons vu en (50), afin de mettre à jour un des nœuds fondamentaux de la pièce, à savoir que le personnage féminin terrorise ses employés. 
Une deuxième possibilité concerne la mise en valeur du mot interrogatif. La valeur rhématique apparaît nettement avec un mot interrogatif frontal, dans le schéma mot interrogatif SV, lorsque le reste de la phrase peut se déduire du contexte ou de la situation :

52. André: On nous a rendu nos courses.

Un gros temps.

Laurence : Comment ils ont eu notre adresse?

André : Je ne sais, moi...

Laurence: Comment ils ont fait pour nous retrouver? (CoDiF, Momo)

Dans les corpus à l'oral spontané, l'emploi de comment en position frontale ne semble pas forcément aussi rhématique :

53. ML666AMI eh mon copain il a perdu 5 kilos

ML666 bah depuis qu'il a commencé ou

ML666AMI non un- un autre un de mes copains

ML666 ah [...] non parce que j'allais dire là celui qu'on ramène là il est déjà pas bien gros

ML666AMI il est toujours euh

ML666 alors il $l^{5}$ va s'envoler

ML666AMI il est toujours ailleurs euh ah il fait quoi je m'en rappelle plus euh

ML666 comment tu peux te faire autant de copains aussi aussi plus rapid- aussi vite?

ML666AMI bah je suis j'ai une équipe hein

(Eslo 2, Repas)

Dans le corpus théâtral, le dialogue (ex. 52) cible un problème (mystère de l'intrusion d'un personnage dans un appartement) alors que c'est beaucoup plus diffus à l'oral spontané : la conversation porte plutôt sur la perte de kilos d'un copain et passe soudain au fait que le locuteur ML666AMI a apparemment plusieurs copains. Le dialogue théâtral pointe à l'aide de comment un élément particulier qui revêt une importance rhématique et dramaturgique, à la différence du dialogue à l'oral spontané.

Est-ce que certaines interrogatives ont une structure qui favorise l'accentuation du mot interrogatif?

Cet aspect a été relevé par plusieurs auteurs pour les interrogatives in situ :

54. Laurence : Elle s'appelle comment? (CoDiF, Momo)

${ }^{5}$ Un copain de ML666AMI. 
Ainsi Coveney (1997) distingue dans ces interrogatives une valeur pragmatique particulière, empreinte d'une forte valeur rhématique par rapport au reste de l'énoncé appartenant au connu (cf. également Cheng et Rooryck (2000)). Dans l'énoncé suivant, l'adverbe interrogatif où porte sur un point nouveau alors que le reste de l'énoncé appartient au connu, comme le montre le démonstratif ça:

55. bah vous ferez ça où vous ferez quoi (Eslo 2, Repas)

Mais, selon Beyssade (2007:188) et Mathieu 2009, le reste de l'énoncé n'appartient pas forcément au connu et la présupposition d'existence de l'événement dans ce type de question n'est pas toujours évidente à déceler (cf. exemples 34 et 35).

Les clivées permettent de mettre en valeur le mot en $q u$ interrogatif (cf. ex 5-8) :

56. et c'est quand que tu vas me le redonner? (Eslo 2, Repas)

Dans les différents cas répertoriés, on note une valeur claire d'insistance mais ce type d'interrogatives n'apparaît pas dans les pièces de théâtre consultées, à part celle de Xavier Durringer qui met en scène des personnages issus d'un milieu social modeste (1 seul exemple) :

57. BEN.- C'était quelle guerre ton père qu'il a fait? (CoDiF, La Quille)

Peut-être sont-elles considérées comme trop familières. On peut relever en revanche des structures interrogatives qui sont des clivées inabouties :

58. Claire: C'est comme ça dans tous les couples! Au bout d'un certain temps, c'est plus pareil. Mais c'est pas pour ça qu'on se quitte?

Axelle : Alors c'est pour quoi?

Claire : Pour tout un tas de raisons... c'est compliqué (CoDiF, Un petit jeu sans conséquence)

La réplique d'Axelle se comprend comme :

Alors c'est pour quoi qu'on se quitte?

Nous avons relevé également une interrogative avec est-ce que et un mot interrogatif différent de que :

59. Pas de place pour les poussettes ! Hein ? Où est-ce que je les mets les poussettes?! Hein, Monsieur l'Indien? (CoDiF, Cochons d'Inde)

traduisant ici un énervement chez le locuteur. A l'oral, cette structure semble également empreinte d'une valeur rhématique, 
ce qui pourrait montrer dans ce cas que la structure en est-ce que comporte une certaine valeur d'insistance :

60. WZ384 tu nous as ramené ta copie d'histoire?

WZ384FIE est-ce que je l'ai ramenée? est-ce que je l'ai ramenée ? où est-ce que je l'ai mise ma copie d'histoire?

WZ384FIL c'est bon les marrons

WZ384FIE oui je l'ai ramenée (Eslo 2, Repas)

Troisièmement, l'acte illocutoire lui-même peut être accentué. C'est ainsi que nous expliquerons la surreprésentation de la structure est-ce que dans le discours théâtral. On peut faire l'hypothèse qu'à l'oral représenté, ce marquage de l'interrogation grâce à est-ce que met clairement en valeur qu'il s'agit d'une interrogative, soulignant l'acte d'énonciation effectuée. On trouve un trait équivalent avec les exclamatives construites à l'aide d'un mot en qu- (quel, comme, qu'est-ce que, que, etc.), peu nombreuses à l'oral spontané (cf. Morel et Danon-Boileau (1998)), mais régulières dans le théâtre, y compris dans les pièces de théâtre considérées pour cette étude :

61. Qu'est-ce que je suis contente... (CoDiF, Momo)

62. Laurence : Quelle histoire! (CoDiF, Comme s'il en pleuvait)

Sans doute s'agit-il de souligner le marquage de la modalité retenue (interrogation, exclamation, $v s$ assertion) ainsi que l'acte illocutoire proféré.

\section{Conclusion}

Nous voyons ainsi d'une part que les interrogatives partielles dans les pièces de théâtre considérées reflètent les interrogatives partielles propres à la langue parlée - nous en retrouvons bien les schémas les plus courants - mais qu'elles diffèrent par leurs fréquences de celles relevées habituellement dans les corpus oraux spontanés et informels : les interrogatives partielles ont pour fonction, dans ce type de discours dramatique, de favoriser la concentration des effets, en facilitant l'enchaînement des répliques, en produisant un resserrement du dialogue et en mettant en valeur le mot interrogatif ou l'acte illocutoire qui découle de l'interrogation.

L'hypothèse parfois avancée est que les structures prononcées par des personnages à la parlure «populaire» pourraient 
correspondre à «des faits attestés comme courants dans la langue parlée » (Favart 2009: 303), «populaire» pouvant être «assimilé à langue orale» (Dufiet et Petitjean 2013). L'observation du discours théâtral dans les pièces contemporaines du corpus CoDiF (théâtre de Sébastien Thiéry, Jean Dell et Gérald Sibleyras) montre que les personnages représentant la classe moyenne (sans être dotés d'une identité populaire, contrairement aux personnages des pièces de Durringer dont nous avons sélectionné une pièce, La Quille) épousent bien les formes attestées des interrogatives dans la langue parlée, sans que l'on puisse évoquer une «parlure populaire », mais que néanmoins des différences demeurent, dues au discours dramatique qui impose ou privilégie certaines structures.

\section{Bibliographie}

Adli A. 2015, «What you like is not what you do: Acceptability and frequency in syntactic variation », in A. Adli, M. G. García, \& G. Kaufmann (éds), Variation in language: System- and Usage- based Approaches, Berlin/Boston, Walter De Gruyter, $\mathrm{p}$. 173-200.

Beyssade C. 2007, «La structure de l'information dans les questions: quelques remarques sur la diversité des formes interrogatives en français », Linx, 55, p. 173-193.

Branca-Rosoff S., Fleury S., Lefeuvre F., Pires M. 2012, «Discours sur la ville. Présentation du Corpus de Français Parlé Parisien des années $2000 »($ CFPP2000), http://cfpp2000.univparis3.fr/CFPP2000.pdf

Cheng L. L.S. et Rooryck J. 2000, "Licensing wh-in-situ", Syntax 3, 1, p. 1-19.

Coveney A. 1997, «L'approche variationniste et la description de la grammaire du français : le cas des interrogative $»$ s. Langue française $\mathrm{n}^{\circ} 115$, p. 88-100.

Dufiet J.-P. et Petitjean A. 2013, Approches linguistiques des textes dramatiques, Paris, Classiques Garnier.

Eshkol-Taravella I., Baude O., Maurel D., Hriba L., Dugua C., Tellier I. 2012, «Un grand corpus oral « disponible » : le corpus d'Orléans 1968-2012, in Ressources linguistiques libres, TAL, volume $52, \mathrm{n}^{\circ} 3 / 2011$, p. 17-46. 
Favart F. 2009, La représentation de l'«oralité poplaire »dans quelques romans du second XX siècle (1966-2006), Thèse de doctorat, sous la direction de Françoise Gadet et d'Enrica Galazzi, Atelier National de reproduction des thèses (ANRT).

Guryev A. 2017, La forme des interrogatives dans le Corpus suisse de SMS en français : étude multidimensionnelle, Thèse de doctorat en co-tutelle, Universités de Neuchâtel et de la Sorbonne Nouvelle, sous la direction de M.-J. Béguelin et de F. Lefeuvre.

Kerbrat-Orecchioni C. 1992, Les interactions verbales, Paris, Armand Colin.

Koch P. \& Oesterreicher W. 1985, «Sprache der Nähe Sprache der Distanz. Mündlichkeit und Schriftlichkeit im Spannungsfeld von Sprachtheorie und Sprachgeschichte », in Romanistisches Jahrbuch, 36. Berlin / New York:Walter de Gruyter, p.15-43.

Koch P. \& Oesterreicher W. 2001, «Gesprochene Sprache und geschriebene Sprache / Langage parlé et langage écrit ». Lexicon des Romanistischen Linguistik 1/2. Tübingen, Max Niemayer Verlag, p. 584-627.

Larthomas P. 1995 ( $1^{\text {ère }}$ édition : 1982), Le Langage dramatique, Paris, PUF.

Le Goffic P. 1993, Grammaire de la phrase française, Hachette sup, Paris.

Le Goffic P. 1997, «Forme et place du sujet dans l'interrogation partielle », Fuchs (éd.), La place du sujet en français contemporain, Louvain-la-neuve, Duculot, p. 15-42.

Lefeuvre, F. 2006, Quoi de neuf sur quoi? Etude morphosyntaxique du mot quoi. Rennes : Presses Universitaires de Rennes.

Lefeuvre F. 2015, «Analyse grammaticale et sur corpus de l'expression c'est quoi (?)», La dia-variation en français actuel. Études sur corpus, approches croisées et ouvrages de référence, Dostie et Hadermann (éds), Bruxelles, Peter Lang, 39-62.

Lefeuvre F. 2016, «Les énoncés averbaux autonomes : approche syntaxique et discursive », La phrase autonome. Théorie et manifestations, (Anscombre J.-C., B. Darbord, A. Oddo, C. Garcia de Lucas eds), Bruxelles, Peter Lang, collection Gramm-R, p. 73-87. 
Lefeuvre F. 2018, «Les interrogatives averbales: le cas de "proforme interrogative + ça" », Les interrogations (Béguelin et Coveney éds), Bruxelles, Peter Lang, p. 183-208.

Lefeuvre F. et Rossi-Gensane N. 2015, «Interrogation », Fiche FRACOV (répertoire grammatical) (http://www.univparis3.fr/index-des-fiches227311.kjsp?RH=1373703153287)

Lefeuvre F. et Parussa G, 2020: «L'oral représenté en diachronie et synchronie: Que nous apprend-il sur l'oral spontané ? »Langages $n^{\circ}$ 217, F. Lefeuvre et G. Parussa eds.

Lefeuvre F. et Tanguy N. 2012, "La représentation de l'oral dans les dialogues dramaturgiques : les énoncés averbaux »" (Quand les genres de discours provoquent la grammaire... et réciproquement, Despierres et Krazem (éds), Condé-surNoireau, Lambert-Lucas, p. 171-186.

Mathieu E. 2009, «Les questions en français : Micro-et macrovariation ». In France Martineau, Raymond Mougeon, Terry Nadasdi \& Mireille Tremblay (éds), Le français d'ici: Études linguistiques et sociolinguistique de la variation, Toronto, $\mathrm{ON}$ : Éditions du GREF, p. 37-66.

Morel M. et Danon-Boileau L. 1998, Grammaire de l'intonation, Paris, Ophrys.

Petitjean A. 2020, «La représentation de l'oral dans les textes dramatiques contemporains », Langages $\mathrm{n}^{\circ} 217$.

Philippe G. 2008, «Registres, appareils formels et patrons » in L. Gaudin-Bordes et G. Salvan (éds), Les registres, enjeux stylistiques et visées pragmatiques, Louvain-La-Neuve, Bruylant Academia, p. 27-37.

Quillard V. 2000, Interroger en français parlé: études syntaxique, pragmatique et sociolinguistique, Thèse de doctorat, Université de Tours, France.

Riegel M., Pellat J.-C., Rioul R. 2009, Grammaire méthodique du français, Paris, PUF, Quadrige.

Scherer J. 1986, La Dramaturgie classique en France, Paris, Nizet.

Wilmet M. 2003, Grammaire méthodique du français, Bruxelles, Duculot.

\section{Corpus}


Corpus CoDiF : Corpus de dialogues en français (Lefeuvre \& Parussa, en cours de constitution)

CFPP2000 (2008-): Corpus de Français Parlé Parisien. http://cfpp2000.univ-paris3.fr/. Sonia Branca-Rosoff, Serge Fleury, Florence Lefeuvre et Matthew Pires. 2012. Discours sur la ville. Présentation du Corpus de Français Parlé Parisien des années 2000 (CFPP2000).

Corpus tiré de Greco, Luca (2002) Interaction, contexte et cognition. Les pratiques de description et de catégorisation de la douleur dans les appels au 15. Thèse de Doctorat, Paris, EHESS.

Corpus scolaire Gomila, Le discours métalinguistique de la classe de lecture, http://syled.univ-paris3.fr/ressources/corpusCDMCL2007/corpus-Paris-3 mai_2010.pdf

Eslo 2 http://eslo.huma-num.fr (sous-corpus repas) 